\title{
Thermal and chemical equilibration of hadronic matter *
}

\begin{abstract}
E. L. Bratkovskaya, W. Cassing, C. Greiner, M. Effenberger, U. Mosel and A. Sibirtsev
Institut für Theoretische Physik, Universität Giessen, 35392 Giessen, Germany

We study thermal and chemical equilibration in 'infinite' hadron matter as well as in finite size relativistic nucleus-nucleus collisions using a BUU cascade transport model with resonance and string degrees-of-freedom. The 'infinite' hadron matter is simulated within a cubic box employing periodic boundary conditions. The various equilibration times depend on baryon density and energy density and are much shorter for particles consisting of light quarks then for particles including strangeness. For kaons and antikaons the chemical equilibration time is found to be larger than $\simeq 40 \mathrm{fm} / \mathrm{c}$ for all baryon and energy densities considered. The inclusion of continuum excitations, i.e. hadron 'strings', leads to a limiting temperature of $T_{s} \simeq 150 \mathrm{MeV}$.
\end{abstract}

\section{INTRODUCTION}

Nucleus-nucleus collisions at relativistic and ultrarelativistic energies are studied experimentally and theoretically to obtain information about the properties of hadrons at high density and/or temperature as well as about the phase transition to a new state of matter, the quark-gluon plasma (QGP). In the latter deconfined partons are the essential degrees of freedom that resolve the underlying structure of hadrons [1]. Whereas the early 'bigbang' of the universe most likely evolved through steps of kinetic and chemical equilibrium, the laboratory 'tiny bangs' proceed through phase-space configurations that initially are far from an equilibrium phase and then evolve by fast expansion. These 'specific initial conditions' - on the theoretical side - have lead to a rapid development of nonequilibrium quantum field theory and nonequilibribrium kinetic theory [2, 3]. Presently, semiclassical transport models are widely used as approximate solutions to these theories and practically are an essential ingredient in the experimental data analysis. For recent reviews we refer the reader to Refs. [4 6 ].

On the other hand, many observables from strongly interacting systems are dominated by many-body phase space such that spectra and abundances look 'thermal'. It is thus tempting to characterize the experimental observables by global thermodynamical quantities like 'temperature', chemical potentials or entropy [1]. We note, that even the use of macroscopic models like hydrodynamics [12,13] employs as basic assumption the concept of local thermal and chemical equilibrium. The crucial question, however, how and on what timescales a global thermodynamic equilibrium can be achieved, is presently a matter of debate. Thus nonequilibrium approaches have been used in the past to address the problem of timescales associated to global or local equilibration [14 21. In

\footnotetext{
${ }^{*}$ Work supported by BMBF, GSI Darmstadt and DFG.
} 
view of the increasing 'popularity' of thermodynamic analyses a thorough microscopic reanalysis of this questions appears necessary especially for nucleus-nucleus collisions at ultrarelativistic energies that aim at 'detecting' a phase transition to the QGP.

In this contribution we discuss equilibration phenomena in 'infinite' hadronic matter using a microscopic transport model that contains both hadron resonance and string degrees-of-freedom. With this investigation we want to provide insight into the reaction dynamics by the use of cascade-like models and also point out some of their limitations. The 'infinite' hadronic matter is modelled by initializing the system solely by nucleonic degrees of freedom through a fixed baryon density and energy density, while confining it to a cubic box and imposing periodic boundary conditions during the propagation in time.

\section{EQUILIBRATION AND LIMITING TEMPERATURE}

To investigate the equilibration phenomena addressed above we perform microscopic calculations using the Boltzmann-Uehling-Uhlenbeck (BUU) model of Refs. [23, 24]. This model is based on the resonance concept of nucleon-nucleon and meson-nucleon interactions at low invariant energy $\sqrt{s}$ [22], adopting all resonance parameters from the Manley analysis [25]. The high energy collisions - above $\sqrt{s}=2.6 \mathrm{GeV}$ for baryon-baryon collisions and $\sqrt{s}=2.2 \mathrm{GeV}$ for meson-baryon collisions - are described by the LUND string fragmentation model FRITIOF [26]. This aspect is similar to that used in the HSD approach [6,27 29] and the UrQMD code [5]. For a detailed description of the underlying model at low energy we refer the reader to Ref. [24].

\subsection{A box with periodic boundary conditions}

In order to study 'infinite' hadronic matter problems we confine the particles in a cubic box with periodic boundary conditions for their propagation similar to a recent box calculation within the UrQMD model [18]. We specify the initial conditions, i.e. baryon density $\rho$, strange particle density $\rho_{S}$ and energy density $\varepsilon$ as follows: first the initial system is fixed to $N_{p}=80$ protons and $N_{n}=80$ neutrons, which are randomly distributed in a cubic box of volume $V$. The 3 -momenta $\vec{p}_{i}$ of the nucleons in a first step are randomly distributed inside a Fermi-sphere of radius $p_{F}=0.26 \mathrm{GeV} / \mathrm{c}$ (at $\rho_{0}$ ) and in a second step boosted by $\pm \beta_{c m}$ by a proper Lorentz transformation. Thus the initial baryon density $\rho$ is fixed as $\rho=A / V, A=N_{p}+N_{n}$. The strange particle density is set to zero as in related heavy-ion experiments while the energy density is defined as $\varepsilon=E / V$, where $E$ is the total energy of all nucleons

$E=\sum_{i}^{A} \sqrt{p_{i}^{2}+m_{N}^{2}}$

The boost velocity $\beta_{c m}$ is related to the initial energy density $\varepsilon$ (excluding Fermi motion) as

$\beta_{c m}=\sqrt{1-\frac{\rho^{2} m_{N}^{2}}{\varepsilon^{2}}}$

using $\varepsilon=\gamma_{c m} \rho m_{N}$ with $\gamma_{c m}=1 / \sqrt{1-\beta_{c m}^{2}}$. Recall that $\rho_{0} m_{N} \simeq 0.15 \mathrm{GeV} / \mathrm{fm}^{3}$ so that an energy density $\varepsilon \simeq 1.5 \mathrm{GeV} / \mathrm{fm}^{3}$ at density $\rho_{0}$ corresponds to $\gamma_{c m} \simeq 10$, i.e. the SPS 
energy $T_{l a b} \simeq 185 \mathrm{~A} \cdot \mathrm{GeV}$. We thus start with a 'true' nonequilibrium situation in order to mimique the initial stage in a relativistic heavy-ion collision. The initial phase represents two interpenetrating, (ideally) infinitely extended fluids of cold nuclear matter.

We now propagate all particles in the box in the cascade mode (without mean-field potentials) using periodic boundary conditions, i.e. particles moving out of the box are reinserted at the opposite side with the same momentum. The phase-space distribution of particles then can change due to elastic collisions, resonance and string production and their decays to mesons and baryons again. We recall that we include all baryon resonances up to an invariant mass of $2 \mathrm{GeV}$ and meson resonances up to the $\phi$-meson. According to the initial conditions for $\varepsilon$ and $\rho$ the factor $\gamma_{c m}$ determines if strings are excited in the very first collisions. This is the case for $\gamma_{c m}>1.4$ where the early equlibration stages are dominated by string formation and decay.

\subsection{Chemical equilibration}

Figure 11 shows the time evolution of the various particle abundances (nucleons $N$, $\Delta, \Lambda, \pi$ and $K^{+}$mesons) for density $\rho=\rho_{0}$ (left panel) at energy density $\varepsilon=0.22$ $\mathrm{GeV} / \mathrm{fm}^{3}$ and for $\rho=3 \rho_{0}$ (right panel) at $\varepsilon=0.66 \mathrm{GeV} / \mathrm{fm}^{3}$. These initial conditions correspond to bombarding energy $T_{l a b}$ per nucleon of roughly $2 \mathrm{~A} \cdot \mathrm{GeV}$. In Fig. 1 we count all particles which are 'hadronized', i.e. produced by string decay after a formation time of $\tau_{F}=0.8 \mathrm{fm} / \mathrm{c}$ in their rest frame.
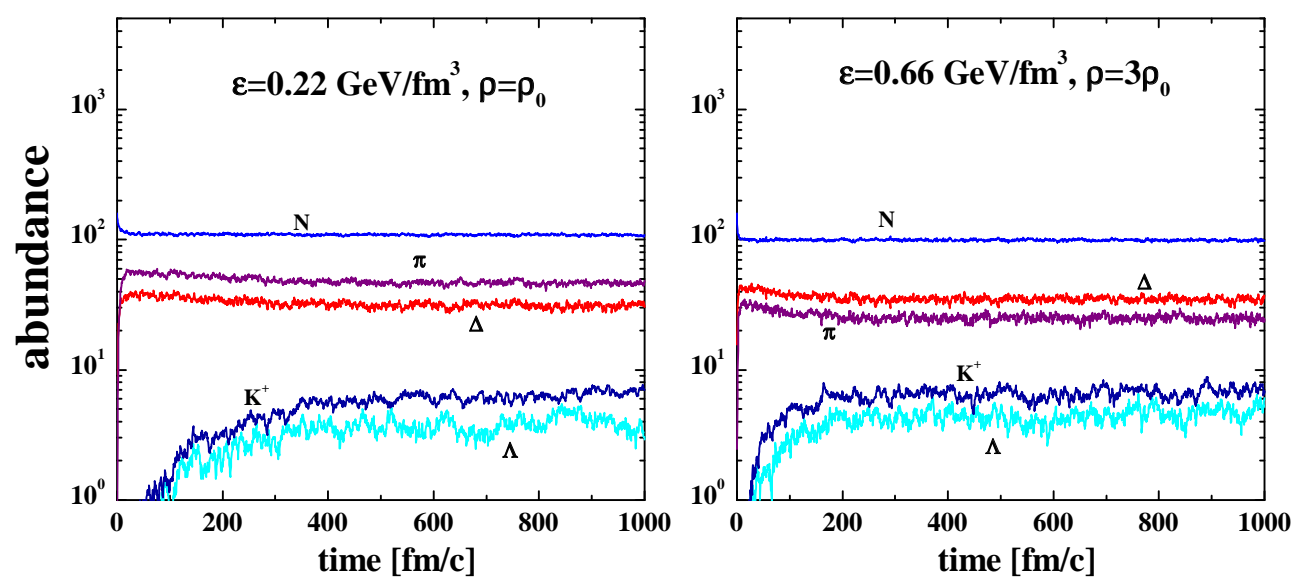

Figure 1. Time evolution of the various particle abundances (nucleons $N, \Delta, \Lambda, \pi$ and $K^{+}$mesons) for density $\rho=\rho_{0}$ (left panel) at energy density $\varepsilon=0.22 \mathrm{GeV} / \mathrm{fm}^{3}$ and for $\rho=3 \rho_{0}$ (right panel) at $\varepsilon=0.66 \mathrm{GeV} / \mathrm{fm}^{3}$.

After several fm/c the number of nucleons decreases due to inelastic collisions that produce either baryon resonances or additional mesons. The number of $\Delta$-resonances grows up to a maximum in a few fm/c, since a lot of $\Delta$ 's are produced in the first $N N$ collisions; their number subsequently decreases with time due to their decay and excitation of further resonances or due to reabsorption. The numbers of $\pi$ 's and $\eta$ 's increase very fast and reach the equilibrium value within a few $\mathrm{fm} / \mathrm{c}$ whereas the strange particles 
$\left(K^{+}, K^{-}, \Lambda\right)$ require a much longer time for equilibration.

For the higher energies the initial particle production proceeds via the formation and decay of string excitations. This leads in particular to a very early onset of strange particles (mainly kaons and hyperons) within the first fm/c either due to the initial strings or due to secondary or ternary baryon-baryon, meson-baryon and meson-meson induced string-like interactions. In Ref. [29] it was shown that these early secondary and ternary reactions can contribute up to about $50 \%$ of the total strange particles obtained in a $\mathrm{Pb}+\mathrm{Pb}$ reaction at CERN SPS energies and thus explain the factor of 2 in the observed relative strangeness enhancement compared to $\mathrm{p}+\mathrm{p}$ reactions. This, however, does not imply that chemical equilibrium for the dominant strange particles has been achieved in this reaction, as our analysis clearly shows. In the later stages, when the system has become, more or less, isotropic in momentum space, strange particles can only be further produced by low energy hadronic reactions, which, however, have a considerable threshold and are thus strongly suppressed. This explains the long chemical equilibration times for the strange particles first demonstrated by Koch, Müller and Rafelski [14].

In order to define an overall chemical equilibration time we perform a fit to the particle abundances $N(t)$ for pions and kaons as

$N(t)=N_{e q}\left(1-\exp \left(-t / \tau_{e q}\right)\right)$

where $N_{e q}$ is the equilibrium limit. The equilibration time $\tau_{e q}$ thus corresponds to the time $t$ when $\simeq 63 \%$ of $N_{e q}$ is achieved.

Figure 2 shows the equilibration time $\tau_{e q}$ versus energy density for $\pi$ and $K^{+}$mesons at different baryon densities of $1 / 3 \rho_{0}, \rho_{0}, 3 \rho_{0}$ and $6 \rho_{0}$. We find that the equilibration time for pions scales as $\tau_{e q}^{\pi} \sim 1 / \rho$ or $\Gamma_{\pi} \sim \rho$, thus we present the curve only for baryon density $\rho_{0}$. Whereas $\tau_{e q}^{\pi}$ slowly grows with energy-density, $\tau_{e q}^{K}$ falls steeply with $\varepsilon$. This marked difference is due to the fact that, on one hand, the kaon production rate increases dramatically with $\sqrt{s}$ whereas that of the pions, on the other hand, is more flat. With increasing energy thus more strange particles are produced through strings especially from the primary collisions with high $\sqrt{s}$ and the chemical equilibration is achieved faster.

In Fig. 2 we have considered an 'ideal' situation, i.e. hadron matter at fixed energy and baryon density. In realistic heavy-ion collisions the system goes through the different stages due to interactions and expansion. However, as follows from Fig. 2, the equilibration time for strangeness is larger than $40 \mathrm{fm} / \mathrm{c}$ for all energy and baryon densities. Thus in realistic nucleus-nucleus collisions the chemical equilibration of strange particles requires also a time above $40 \mathrm{fm} / \mathrm{c}$ which is considerably larger than the actual reaction time of a few $10 \mathrm{fm} / \mathrm{c}$ or less [30].

The particle abundances used to extract $\tau_{e q}$ in Fig. 2 have been calculated without any in-medium potentials. In fact, the introduction of attractive potentials (especially for $\mathrm{K}^{-}$) will lower the hadronic thresholds and thus increase the scattering rate between strange and nonstrange hadrons, whereas the $K^{+}$feels some repulsive potential and the trend goes in the opposite way. According to our calculations such in-medium modifications (in line with Ref. [6]) give a correction to the $K^{+}$equilibration times by atmost $10 \%$ and shortens the $K^{-}$equilibration times up to $20 \%$ at density $\rho_{0}$. 


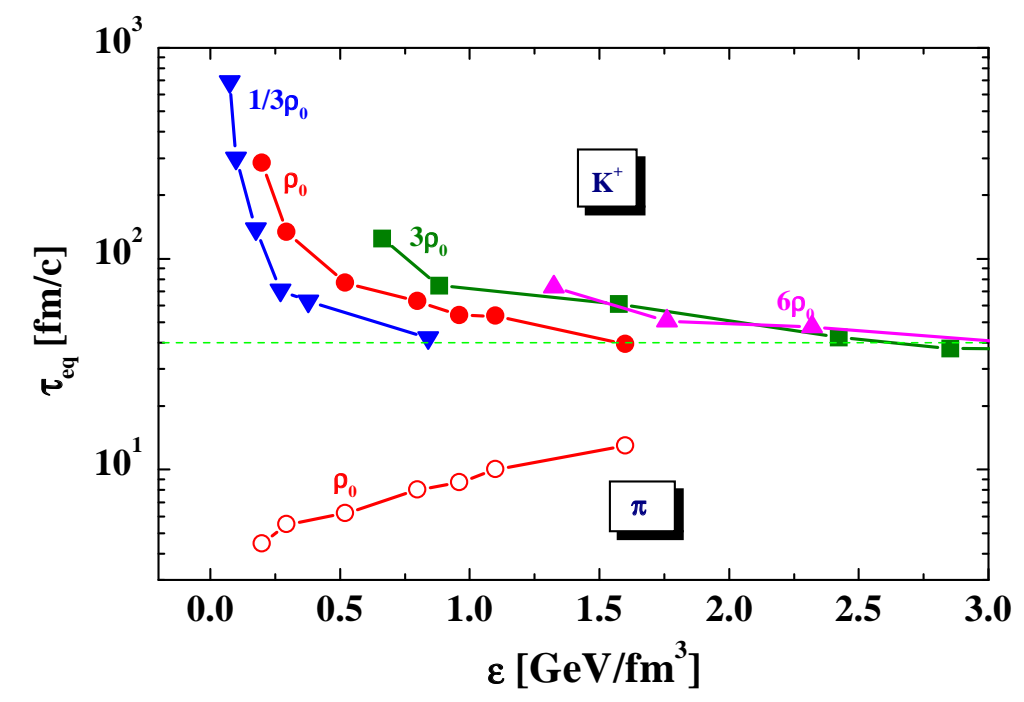

Figure 2. Equilibration time $\tau_{e q}$ versus energy density $\varepsilon$ for $\pi$ and $K^{+}$mesons at different baryon densities $1 / 3 \rho_{0}, \rho_{0}, 3 \rho_{0}$ and $6 \rho_{0}$.

\subsection{Thermal equilibration and limiting temperature}

In this subsection we investigate the approach to thermal equilibration. For the equilibrated system we can extract a temperature $T$ by fitting the particle spectra with the Boltzmann distribution

$\frac{d^{3} N_{i}}{d p^{3}} \sim \exp \left(-E_{i} / T\right)$

where $E_{i}=\sqrt{p_{i}^{2}+m_{i}^{2}}$ is the energy of particle $i$. We note that at the temperatures of interest here, the Bose and Fermi distributions are practically identical to a Boltzmann distribution. We find that in equilibrium the spectra of all particles can be characterized by one single temperature $T$ [30].

In the left panel of Fig. 3 we display the temperature $T$ versus energy density $\varepsilon$ for different baryon densities $\rho: 1 / 3 \rho_{0}$ (open down triangles), $\rho_{0}$ (full squares), $3 \rho_{0}$ (full dots), $6 \rho_{0}$ (full up triangles). In order to compare calculations for different baryon densities we have subtracted the baryon energy density at rest, i.e. $\simeq m_{N} \rho$ (except for Fermi motion). As seen from Fig. 3 the temperature grows with energy density up to a limiting value reminiscent of a 'Hagedorn' temperature [31]. From our detailed investigations we obtain for the limiting temperature $T_{s} \simeq 150 \pm 5 \mathrm{MeV}$ which practically does not depend on baryon density. Such a singular behavior of $\varepsilon(T)$ for $T \simeq T_{s}$ has also been found in the box calculations in Ref. [18] for $\rho=\rho_{0}$. Our limiting temperature is slightly higher than that in Ref. 18 $\left(T_{s}=130 \pm 10 \mathrm{MeV}\right)$ due to the different number of degrees of freedom; the model [18] contains more resonances and uses a different threshold for string excitations. Thus, there is some phenomenological sensitivity to the hadronic zoo of particles and string thresholds employed in the model.

In order to investigate the equilibrium behavior of hadron matter we also compare our transport (box) calculations with a simple Statistical Model (SM) for an Ideal Hadron Gas 

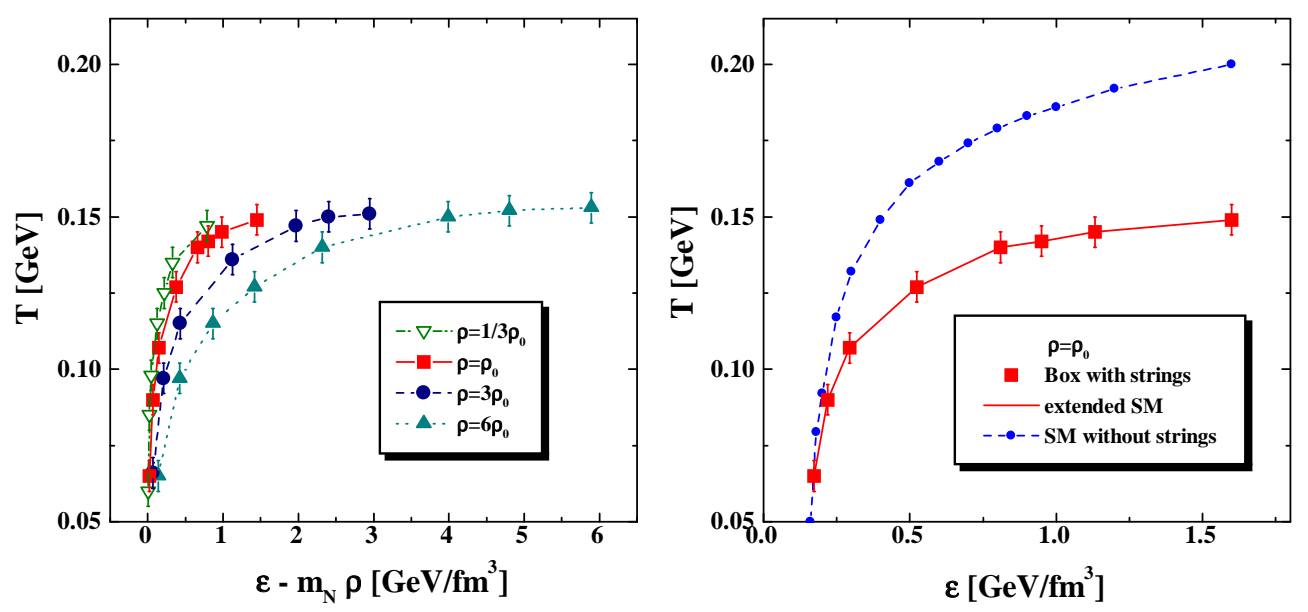

Figure 3. Left panel: equilibrium temperature $T$ versus the energy density $\varepsilon-m_{N} \rho$ for different baryon densities $\rho: 1 / 3 \rho_{0}$ (open down triangles), $\rho_{0}$ (full squares), $3 \rho_{0}$ (full dots), $6 \rho_{0}$ (full up triangles). Right panel: equilibrium temperature $T$ versus the energy density for baryon density $\rho=\rho_{0}$. The full dots correspond to the statistical model (SM) without strings, the full squares show our box calculations including string degrees of freedom, while the solid line shows the result from the extended SM including a Hagedorn mass spectrum for strings.

where the system is described by a grand canonical ensemble of non-interacting fermions and bosons in equilibrium at temperature $T$. All baryon and meson species considered in the transport model [23] also have been included in the statistical model [30].

Within the SM we find that the temperature increases continuously with energy density since the continuum excitations, i.e. the string degrees of freedom, are not included (full dots in the right panel of Fig. 3), whereas the box calculation with strings gives the limiting temperature (full squares in Fig. 3). Both curves in Fig. 3 have been calculated for density $\rho_{0}$.

To reproduce qualitatively our box result within the SM we have to include continuum excitations in the statistical model, i.e. a Hagedorn mass spectrum for strings [31] (for details see [30]). For the 'Hagedorn' temperature $T_{H}$ we use the temperature $T_{s}$ as obtained from the box calculations, i.e. $T_{H}=T_{s} \simeq 150 \mathrm{MeV}$. As seen in right panel of Fig. 3 we achieve agreement of the extended SM and our box calculations. However, we point out that the limiting temperature $T_{s}$ from our string model involves somewhat different physics assumptions than the Hagedorn model at temperature $T_{H} . T_{s}$ should not really be identified with the 'Hagedorn' temperature $T_{H}$, though close similarities exist. In the Hagedorn picture and for temperatures close to $T_{H}$ the abundance of 'normal' hadrons or known resonances stays constant with increasing energy density whereas the number and energy density of the (hypothetical) bootstrap excitations diverges for $T \rightarrow T_{H}$. The Hagedorn model thus assumes 'particles' of mass $m \rightarrow \infty$ to be populated for $T \rightarrow T_{H}$, that dynamically can be formed in collisions of high mass hadrons for $t \rightarrow \infty$. In contrast, our string model does not include energetic string-string interactions that might produce more massive strings. 


\section{SUMMARY}

In this contribution we have performed a systematic study of equilibration phenomena and equilibrium properties of 'infinite' hadronic matter as well as of relativistic nucleus-nucleus collisions using a BUU transport model that contains resonance and string degrees-of-freedom. The 'infinite' hadron matter is modelled by initializing the system at fixed baryon density, strange density and energy density by confining it in a cubic box with periodic boundary conditions 30.

We have shown that the equilibration times $\tau_{e q}$ for different particles depend on baryon density and energy density. The time $\tau_{e q}$ for non-strange particles is much shorter than for particles including strangeness; for kaons and antikaons the equilibration time is found to be larger than $\simeq 40 \mathrm{fm} / \mathrm{c}$ for all baryon and energy densities considered. The overall abundance of the dominant strange particles (kaons and $\Lambda$ 's) being produced and obtained within the BUU cascade model for heavy-ion collisions can therefore not be described by assuming a perfect chemical equilibrium as strangeness is typically still undersaturated to a quite large extent. We mention that transport model calculations like ours can describe the yield and spectra of the produced nonstrange hadrons as well as $K^{+}, K^{-}, \Lambda$ yields quite well at SPS energies [6,29]. On the other hand, at AGS energies the measured $K^{+} / \pi^{+}$ ratio in central $\mathrm{Au}+\mathrm{Au}$ collisions is underestimated by about 30\% 32. However, we have to point out that the more exotic strange particles (like the measured antihyperon yields of Ref. [33]) can by far not be explained within such standard hadronic multiple channel reactions. These hadronic data possibly point towards new physics.

We have, furthermore, shown that thermal equilibrium is established quickly, within about $5 \mathrm{fm} / \mathrm{c}$ at SIS energies and samewhat larger times at high energies. The inclusion of continuum excitations, i.e. hadron 'strings', leads to a limiting temperature of $T_{s} \simeq$ $150 \mathrm{MeV}$ in our transport approach which practically does not depend on the baryon density and energy. We have compared our results with the statistical model (SM), which contains the same degrees of freedom and the same spectral functions of particles as our transport model. We found that the limiting temperature behaviour can be reproduced in the statistical model only after including continuum excitations of the Hagedorn type, otherwise the fireball temperature extracted from the particle abundances and spectra is overestimated substantially.

Close to the critical temperature $T_{s}$, the hadronic energy densities can increase to a couple of $\mathrm{GeV} / \mathrm{fm}^{3}$. From lattice QCD calculations one expects that a phase transition to a potentially deconfined QGP state should occur. Referring to the limiting temperature $T_{s} \approx 150 \mathrm{MeV}$ obtained, a QGP should be revealed and clearly distinguished from a hadronic state of matter if one can unambiguously prove the existence of an equilibrated and thermal phase of strongly interacting matter with temperatures exceeding, e.g., 200 $\mathrm{MeV}$. The best candidates are electromagnetic probes, either direct photons or dileptons. On the other hand these are also 'contaminated' by hadronic background and/or preequilibrium physics. So far no thermal electromagnetic source with temperatures larger or equal than $200 \mathrm{MeV}$ has been clearly identified. This might happen at RHIC energies in central $\mathrm{Au}+\mathrm{Au}$ collisions which are expected to be studied soon.

\section{REFERENCES}


1. QUARK MATTER '96, Nucl. Phys. A 610 (1997) 1; QUARK MATTER '97, Nucl. Phys. A 638 (1998) 1; QUARK MATTER '99, Nucl. Phys. A 669 (1999) 1.

2. W. Botermans and R. Malfliet, Phys. Rep. 198 (1990) 115.

3. P.A. Henning, Phys. Rep. 253 (1995) 235.

4. G. Q. Li and C. M. Ko, J. Phys. G 22 (1997) 1673.

5. S. A. Bass et al., Prog. Part. Nucl. Phys. 42 (1998) 279; J. Phys. G 25 (1999) 1859.

6. W. Cassing and E. L. Bratkovskaya, Phys. Rep. 308 (1999) 65.

7. P. Braun-Munzinger, J. Stachel, J.P. Wessels, and N. Xu, Phys. Lett. B 344 (1995) 43; Phys. Lett. B 365 (1996) 1; J. Stachel, Nucl. Phys. A 654 (1999) 119.

8. J. Cleymans and H. Satz, Z. Phys. C 57 (1993) 135.

9. J. Sollfrank, M. Gazdzicki, U. Heinz and J. Rafelski, Z. Phys. C 61 (1994) 659; F. Becattini, M. Gazdzicki and J. Sollfrank, Eur. Phys. J. C 5 (1998) 143.

10. C. Spieles, H. Stöcker and C. Greiner, Eur. Phys. J. C 2 (1998) 351.

11. J. Cleymans, H. Oeschler, and K. Redlich, nucl-th/9809027; J. Phys. G 25 (1999) 281.

12. H. Stöcker and W. Greiner, Phys. Rep. 137 (1986) 277.

13. U. Ornik et al., Phys. Rev. C 54 (1996) 1381; S. Bernard et al., Nucl. Phys. A 605 (1996) 566; J. Sollfrank et al., Phys. Rev. C 55 (1997) 392.

14. P. Koch, B. Müller, and J. Rafelski, Phys. Rep. 142 (1986) 167.

15. W. Cassing, V. Metag, U. Mosel, and K. Niita, Phys. Rep. 188 (1990) 363.

16. A. Lang, B. Blättel, W. Cassing, V. Koch, U. Mosel, and K. Weber, Z. Phys. A 340 (1991) 287.

17. B. Blättel, V. Koch, and U. Mosel, Rep. Progr. Phys. 56 (1993) 1.

18. M. Belkacem, M. Brandstetter, S.A. Bass et al., Phys. Rev. C 58 (1998) 1727.

19. L.V. Bravina, M.I. Gorenstein, M. Belkacem et al., Phys. Lett. B 434 (1998) 379; L.V. Bravina, M. Brandstetter, M.I. Gorenstein et al., J. Phys. G 25 (1999) 351.

20. L.V. Bravina, E.E. Zabrodin, M.I. Gorenstein et al., Phys. Rev. C 60 (1999) 024904.

21. J. Sollfrank, U. Heinz, H. Sorge, N. Xu, Phys. Rev. C 59 (1999) 1637.

22. S. Teis, W. Cassing, M. Effenberger, A. Hombach, U. Mosel, and Gy. Wolf, Z. Phys. A 356 (1997) 421.

23. M. Effenberger, E.L. Bratkovskaya, and U. Mosel, Phys. Rev. C 60 (1999) 044614.

24. M. Effenberger, Ph.D. Thesis, Univ. of Giessen, 1999; http://theorie.physik.unigiessen.de/ftp.htm].

25. D. M. Manley and E. M. Saleski, Phys. Rev. D 45 (1992) 4002.

26. B. Anderson, G. Gustafson and Hong Pi, Z. Phys. C 57 (1993) 485.

27. W. Ehehalt and W. Cassing, Nucl. Phys. A 602 (1996) 449.

28. E. L. Bratkovskaya and W. Cassing, Nucl. Phys. A 619 (1997) 413.

29. J. Geiss, W. Cassing, and C. Greiner, Nucl. Phys. A 644 (1998) 107.

30. E. L. Bratkovskaya, W. Cassing, C. Greiner, M. Effenberger, U. Mosel and A. Sibirtsev, Nucl. Phys. A 675 (2000) 661.

31. R.Hagedorn, Suppl. Novo Cimento 3 (1965) 147; Suppl. Novo Cimento 6 (1965) 311; R.Hagedorn and J. Ranft, Suppl. Novo Cimento 6 (1968) 169.

32. W. Cassing, Nucl. Phys. A 661 (1999) 468c.

33. E. Andersen et al., J. Phys. G 25 (1999) 171; J. Phys. G 25 (1999) 181. 